\title{
A New Approach to Specify Real-Time Behavior of Distributed Systems
}

\author{
R. Mall \\ Dept. of Computer Science and Automation \\ Indian Institute of Science \\ Bangalore, INDIA. \\ e-mail: mal@vigyan.ernet.in
}

\begin{abstract}
Specification of real-time behavior of a system requires a significantly differentapproach compared to specification of the system's non-real-time behavior. Also, a specification formalism should be easy to use, conceptually simple, and should lead to an intuitive understanding about the specified behavior of the system. With theseobjectives, in this paper, we develop an event-based approach to specify the real-time behavior and relevant properties of distributed systems. In our approach, events are considered as the basic entities and a system is specified by constructing various relations among the events. Based on this formalism, a specification language is also presented. A specification in this language can be automatically transformed into equivalent specifications in many existing formalisms (e.g, temporal logic, firstorder logic, etc.) for further analysis. Thus, an eventbased specification can be used as a front-end tool for automatically generating specificationsin many existing formalisms, which can save the trouble of writing complicated formulas in those formalisms.
\end{abstract}

\section{Introduction}

Distributed computer systems are finding increasing use in real-time process control applications. Most of these applications are safety-critical in nature, requiring use of highly reliable computer systems. Building such systems is a nontrivial problem, since the correct behavior of these systems entails not only functional correctness but also timeliness of the results. Thus, any inaccuracy in the specification of the real-time behavior of such systems can lead to costly system failures. Consequently, a suitabletool to formally specify the real-time behavior and the relevant properties of distributed realtime systems is vital to realizing the required performance and reliability levels.

Specification of real-time behavior of systems requires a significantly different approach compared to specification of non-real-timebehavior - as not only the eventual occurrence but also the exact timing of various events becomes important. Further, in case of distributed sys-

\author{
L.M. Patnaik
}

Microprocessor Applications Laboratory, SupercomputerEducation and Res. Centre, and Dept. of Computer Science and Automation Indian Institute of Science Bangalore, INDIA. lalit@vigyan.ernet.in

tems, in addition to precisely specifying the relative timing of various events, distributedconcurrency aspects should also be represented. On the other hand, a specificationformalism should be easy to use, conceptually simple, and should also lead to an intuitive understanding about the system's specified behavior. In this regard, we feel that post hoc introduction of "time" into an existing specification formalism does not lead to natural expression of the real-time behavior of systems. Another motivation for our work is the fact that most of the timing specification tools reported in the literature (e.g., $[2,8]$ ) require writing complicated mathematical expressios and/or knowledge of specialized mathematical theories. Complex specifications often obscure intuitive understanding of a system's specified behavior. In this context, we develop a formal specification method that considersevents as the basic entities. We feel that it is intuitively appealing to express the real-time behavior of systems in terms of event occurrences, as events are essentially markers on a space-time diagram. Real-time behavior of systems is expressed by defining various relations on the events. This method, while expressive enough to specify the rich behavior of distributed realtime systems, is also intuitively appealing and simple to use.

An important characteristicfeature of distributed systems is that the events occur at the spatially separated nodes. Also, a node typically remains unaware of various events occurring at other nodes of the system, unless it specifically receives a message to that end. Our specification method is designed to represent this important behavioral aspect of distributed systems. For this purpose, we consider events as markers in time and space; and use nodes as the basic unit of specification of distributed systems. Based on the developed language, a specification language is also presented. Also, specifications in this language can be automatically transformed into equivalent specifications in many existing formalisms (e.g., temporal logic [2,8], first-order logic [3], etc.) for further analysis. Such automatic transformationcan save the tedium of writing complicated formulas while specifying a system in those formalisms. 
The rest of this paper is organized as follows. Section 2 develops the event-based specification formalism. Based on the developedformalism, section 3 formulates a specification language called TSDS (Timing Specifier of Distributed Systems). Section 4 illustrates the use of the specification language with an example. Section 5 presents a comparison of our approach with the related work. Section 6 concludes this paper.

\section{Event-Based Specification \\ 2.1 Preliminaries}

We considerevents as the basic entities in terms of which the real-timebehavior and the relevant properties of distributed systems are specified. Although it is possible to develop the specification formalism solely in terms of occurrence of events, it is more convenient to define and use actions as derived entities. Predicates will be used to ascribe meaning to events by relating their occurrences to the aspects of behavior of the physical system. However, we will mainly be interested in the events which change the truth value of predicates. Realtime behavior of distributed systems is expressed by first enumerating the significant events associated with each node of the system, and then by constructing certain relationships among the events. This ordering among events is in the tradition of Winskel's event structures [4].

Definition 2.1: Each occurrence of an event marks a point in time and space that is of importance in describing a system's real-time behavior. The set of events (E) associated with a distributed system can be partitioned into the following six classes:

i.) External events (EE): An external event occurs due to the action(s) of the environment on an embedded system.

ii.) Start events (EB): A start event marks the initiation of an action (see def. 2.3).

iii.) Stop events (ES): A stop event marks the completion of an action (see def. 2.3).

iv.) Transition events (ET): A transition event marks the instant at which a change to the truth values of certain formula(s) describing the system behavior occurs. An event marking the transition of a formula $\mathrm{X}$ to true is represented by $(\bullet \mathrm{X})$ and the event marking the time point at which the formula becomes false is represented by $\left({ }^{\circ} \mathrm{X}\right)$.

v.) Notifier events (EN):A notifier event at a node marks a point on time at which the node transmits a message to another node.

vi.) Notification events (EA): A notification event at a node marks a point in time, at which it receives a message from another node.

Thus, $E=E E$ EB UES UET UEN U EA, and
$\mathrm{E}_{\mathrm{i}} \cap \mathrm{E}_{\mathrm{j}}=\Phi, \mathrm{E}_{\mathrm{i}} \neq \mathrm{E}_{\mathrm{j}}, \mathrm{E}_{\mathrm{i}}, \mathrm{E}_{\mathrm{j}} \in\{\mathrm{EE}, \mathrm{EB}, \mathrm{ES}, \mathrm{ET}, \mathrm{EN}, \mathrm{EA}\}$.

An event $e$ can have more than one instance (occurrence). Each time an event occurs (i.e, an instance), it acquires a label from the set of natural numbers $\mathbf{N}$, so that the ith instance of an event $\boldsymbol{e}$ can be uniquely represented by $(e, \mathrm{i}), \mathrm{i} \in \mathrm{N}$. We will use $e$ itself to representall occurrences of that event. To emphasize the fact that events occur at the spatially separated nodes, event names will be prefmed by the names of the nodes at which they occur. It should be noted that the definition of events in our model is significantly different from those in $[7,4]$.

Definition 22 : A node is the basic unit of specification of distributed systems. A node consists of a collection of events (and actions) associated with a common time base, a definition of various relationships among these events (and actions) as well as the relationships of these events (and actions) with those of the other nodes of the system.

Definition 23: Actions are the schedulable entities in distributed computations. Occurrence of each actions represents finite progress made in a computation. Instances of actions can be defined in terms of the corresponding start and stop events.

The names of actions are unique and are usually prefmed by the names of the nodes at which they occur. Like events, an action can occur a number of times. The functions begin and end will be used to project out the start and stop events of an instance of an action. Thus for an action instance $\left(\mathrm{a}_{1}, \mathrm{i}\right)$ the start event is given by begin $\left(\mathrm{a}_{1}, \mathrm{i}\right)$ and the stop event can be represented by end $\left(\mathrm{a}_{1}, \mathrm{i}\right)$.

Definition 2.4 Predicates relate event occurrences to the behavior of the physical system. The value of a predicate can change with time due to the occurrence of transition events.

In the specification of a system, we are particularly interested in the times at which a predicate (describing aspects of systembehavior) becomes true and at which it becomesfalse (i.e. the transition events).

\subsection{Relation Among Events}

Definition 2.5: A temporal precedence relation $(\mathrm{Lt})$ among the events in a distributed system is defined as follows. The relation $\left(e_{1}, \mathrm{i}\right) L_{t}\left(\left(e_{2}, j\right), t_{1}, t_{2}\right)$, implies that the jth instance of the event $e_{2}$ occurs between $t i$ and $t_{2}$ time units after the ith instance of ei occurs.

Definition 2.6 A causal relation $\left(L_{c}\right)$ on events is defined as follow: $\left(e_{1}, i\right) \angle_{c}\left(e_{2}, j\right)$, if the occurrence of the event $\left(e_{1}, i\right)$ somehow causes the event $\left(e_{2}, j\right)$ to occur eventually. Thus, the causal relation $\left(e_{1}, i\right) L_{c}\left(e_{2}, j\right)$ is, in effect, a short form for the relation $\left(e_{1}, i\right)$ $\angle_{t}\left(\left(e_{2}, j\right), 0, \infty\right)$. Two events $\left(e_{1}, \mathrm{i}\right)$ and $\left(e_{2}, j\right)$ are called unrelated (concurrent), if $\left(\mathrm{e}_{1}, \mathrm{i}\right) k_{\mathrm{c}}\left(\mathrm{e}_{2}, \mathrm{j}\right)$ and $\left(\mathrm{e}_{2}, \mathrm{j}\right) k_{\mathrm{c}}\left(\mathrm{e}_{1}, \mathrm{i}\right)$. 


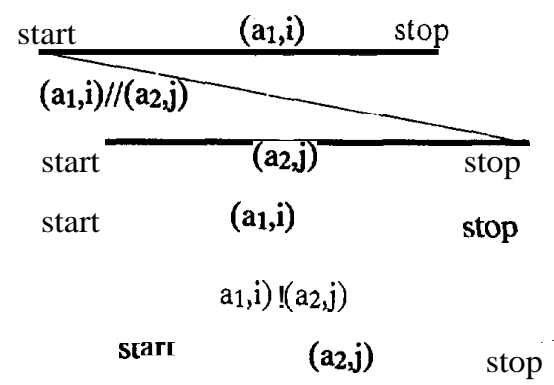

Fig. 1 Relation Among Actions start

$\left(a_{1}, \mathbf{i}\right)$

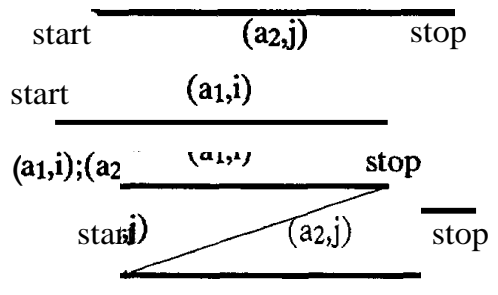

Definition 27: Two events $\left(e_{1}, i\right)$ and $\left(e_{2}, j\right)$ are said to be causally equal (denoted by $\left(e_{1}, i\right)=c\left(e_{2}, j\right)$, iff there existsanevent $\left(e_{3}, k\right)$ such that $\left(e_{3}, k\right) L_{c}\left(e_{1}, i\right),(e 3, k) L_{c}$ $\left(e_{2}, j\right),\left(e_{1}, i\right) \psi_{c}\left(e_{2}, j\right)$, and $\left(e_{2}, j\right) k_{c}\left(e_{1}, i\right)$. Causal equality among actions can be defined in a similar way: two actions $\left(a_{1}, i\right)$ and $\left(a_{2}, j\right)$ are called causally equal $\left(a_{1}, i\right)=c$ $\left(a_{2}, j\right)$, iff begin $\left(a_{1}, i\right)=c$ begin $\left(a_{2}, j\right)$.

Definition 2.8 Two events $\left(e_{1}, \mathrm{i}\right)$ and $\left(e_{2}, j\right)$ are said to be temporally equal $\left(e_{1}, i\right)=t\left(e_{2}, j\right)$, if they are causally equal, and occur at precisely the same time instant. Temporal equality is especially useful in describing synchronous events.

Definition 2.9: Two events $e_{1}$ and $e_{2}$ are said to be in conflict with each other (represented by $e_{1} \# e_{2}$ ), if the occurrence of one event forbids the occurrence of the other. The conflict relation provides a way to describe choices available to a system. Obviously, if $e_{1} L_{c}$ e2, e 2 $L_{\mathrm{c}} \mathrm{e} 3$, and $\mathbf{e}_{1} \# \mathrm{e}_{4}$, then $\mathrm{e}_{3} \# \mathrm{e}_{4}$.

\subsection{Relation among Actions}

The causal dependency among actions can be represented by using the following constructs (also shown in fig. 1$):\left(\mathbf{a}_{1}, \mathbf{i}\right) ;\left(\mathbf{a}_{2}, \mathbf{j}\right)$ is used to denote that the action $\left(a_{2}, \mathrm{j}\right)$ can start only after $\left(\mathrm{a}_{1}, \mathrm{i}\right)$ completes; $\left(\mathrm{a}_{1}, \mathrm{i}\right) / /\left(\mathrm{a}_{2}, \mathrm{j}\right)$ denotes that the action $(a 2, j)$ must finish only after $(a 1, i)$ starts; $\left(a_{1}, i\right) \backslash \backslash\left(a_{2}, j\right)$ denotes that the action $\left(a_{2}, j\right)$ can start only after $\left(a_{1}, i\right)$ starts; and $\left(a_{1}, i\right) !\left(a_{2}, j\right)$ means that action $\left(a_{1}, \mathrm{i}\right)$ must complete before $\left(\mathrm{a}_{2}, \mathrm{j}\right)$ completes.

Two actions are called sequential if any pair of time points in the execution of the two actions can be compared. Two actions are called primitively concurrent, if there is at least a pair of time points between the intervals representing the two actions not comparable. Thus, the actions $\left(a_{1}, i\right)$ and $\left(a_{2}, j\right)$ in $\left(a_{1}, i\right) ;\left(a_{2}, j\right)$ are sequentially dependent, whereas those in $\left(a_{1}, i\right) \backslash \backslash(a t, j),\left(a_{1}, i\right) / /(a 2, j)$ and $\left(\mathrm{a}_{1}, \mathrm{i}\right) !\left(\mathrm{a}_{2}, \mathrm{j}\right)$ are primitively concurrent. Two actions $\left(a_{1}, i\right)$ and $\left(a_{2}, j\right)$ are called totally concurrent (denoted by $\left.\left(a_{1}, \mathrm{i}\right)||\left(a_{2}, j\right)\right)$, if no time point in their execution can be compared.

Definition 2.10 : A computation $(C)$ represents a single run of the specified system. A computation can be rep- resented by a single action $\mathrm{C}$, which can be decomposed into more primitive actions. The start event of the action $\mathrm{C}, \mathrm{e}_{\mathrm{s}}=$ begin( $\mathrm{C}$ ) signals the start of a computation and the stop event of the action $C, e_{e}=\operatorname{end}(C)$ signalsthe end of a computation. Of course, for cyclic and nonterminating executions, the stop event may never occur.

\section{Specification Language TSDS}

In order to facilitate specification of real-time behavior of distributed systems in the event-based model discussed in the previous section, we have developed a language called TSDS (Timing Specifier for Distributed Systems) providinga set of language constructs for this purpose. After enumerating the nodes of the system along with their associated events and actions, the language constructs of TSDS can be used to express the relations among the events. Each construct of the language actually represents a set of relations among the events of the system.

The following are the language constructs of TSDS and the relations they represent:

\section{Events}

If $\left(e_{1}, i\right)$ and $\left(e_{2}, j\right)$ are event instances, then Construct Interpretation

$\left(\mathrm{e}_{1}, \mathrm{i}\right)$ precedes $\left(\mathrm{e}_{2}, \mathrm{j}\right)$ by $\left(\mathrm{t}_{1}, \mathrm{t}_{2}\right) \quad\left(\mathrm{e}_{1}, \mathrm{i}\right) L_{\mathrm{t}}\left(\left(\mathrm{e}_{2}, \mathrm{j}\right), \mathrm{t}_{1}, \mathrm{t}_{2}\right)$

$\left(e_{1}, \mathrm{i}\right)$ succeeds $\left(e_{2}, \mathrm{j}\right)$ by $\left(\mathrm{t}_{1}, \mathrm{t}_{2}\right) \quad\left(\mathrm{e}_{2}, \mathrm{j}\right) \angle_{\mathrm{t}}\left(\left(\mathrm{e}_{1}, \mathrm{i}\right), \mathrm{t}_{1}, \mathrm{t}_{2}\right)$

$\left(e_{1}, i\right)$ causes $\left(e_{2}, j\right)$

$e_{1}$ conflictswith $e_{2}$

$\left(e_{1}, \mathrm{i}\right) \angle_{c}\left(e_{2}, \mathrm{j}\right)$

Actions

If $\left(a_{1}, i\right)$ and $\left(a_{2}, j\right)$ are action instances, then

\begin{tabular}{|c|c|}
\hline Construct & Interpretation \\
\hline$\left(a_{1}, \mathrm{i}\right) ;\left(a_{2}, \mathrm{j}\right)$ & end $\left(a_{1}, i\right) \angle_{c} \operatorname{begin}\left(a_{2}, j\right)$ \\
\hline$\left(a_{1}, \mathrm{i}\right) \backslash \backslash\left(a_{2}, \mathrm{j}\right)$ & $\operatorname{begin}\left(a_{1}, \mathrm{i}\right) \angle_{c} \operatorname{begin}\left(a_{2}, \mathrm{j}\right)$ \\
\hline$\left(a_{1}, \mathrm{i}\right) / /\left(a_{2}, \mathrm{j}\right)$ & $\operatorname{begin}\left(a_{1}, \mathrm{i}\right) \angle_{c}$ end $\left(a_{2}, \mathrm{j}\right)$ \\
\hline$\left(a_{1}, \mathbf{i}\right) !\left(a_{2}, j\right)$ & end $\left(a_{1}, i\right) \angle_{c}$ end $\left(a_{2}, j\right)$ \\
\hline
\end{tabular}

$$
\begin{gathered}
\left(e_{1}, i\right)<t\left(\operatorname{begin}\left(a_{2}, 1\right), t_{1}, t_{1}\right) \\
\operatorname{begin}\left(a_{2}, j\right)<t\left(\operatorname{begin}\left(a_{2}, j+1\right), t_{1}, t_{1}\right)
\end{gathered}
$$




$$
\begin{array}{ll}
\left(a_{1}, \mathrm{i}\right) \operatorname{fork}\left(\left(\mathrm{a}_{2}, \mathrm{j}\right),\left(\mathrm{a}_{3}, \mathrm{k}\right)\right) & \operatorname{end}\left(\mathrm{a}_{1}, \mathrm{i}\right) \angle_{c} \text { begin }\left(\mathrm{a}_{2}, \mathrm{j}\right) \\
& \operatorname{end}\left(\mathrm{a}_{1}, \mathrm{i}\right) \angle_{c} \text { begin }\left(\mathrm{a}_{3}, \mathrm{k}\right) \\
& \text { begin }\left(\mathrm{a}_{2}, \mathrm{j}\right)=\mathrm{t} \text { begin }\left(\mathrm{a}_{3}, \mathrm{k}\right) \\
\left.\left(\mathrm{a}_{1}, \mathrm{i}\right) \text { join }\left(\left(\mathrm{a}_{2}, \mathrm{j}\right),\left(\mathrm{a}_{3}, \mathrm{k}\right)\right)\right) & \left(\mathrm{a}_{1}, \mathrm{i}\right) !\left(\mathrm{a}_{2}, \mathrm{j}\right),\left(\mathrm{a}_{1}, \mathrm{i}\right) !\left(\mathrm{a}_{3}, \mathrm{k}\right) \\
\left(\mathrm{a}_{1}, \mathrm{i}\right) \text { lasts ti } & \text { begin }\left(\mathrm{a}_{1}, \mathrm{i}\right) \angle_{t}\left(\operatorname{end}\left(\mathrm{a}_{1}, \mathrm{i}\right), \mathrm{t}_{1}, \mathrm{t}_{1}\right)
\end{array}
$$

\section{Predicates}

If $\mathbf{P}_{\mathbf{1}}$ and $\mathrm{P}_{\mathbf{2}}$ are predicates describing aspects of behavior of the physical system, then

$$
\begin{array}{ll}
\text { Construct } & \text { Interpretation } \\
\text { Always } \mathrm{Pi} & \bullet \mathrm{P}_{1}={ }_{t} \mathrm{e}_{\mathrm{s}},{ }^{\circ} \mathbf{P}_{1}={ }_{\mathrm{t}} \mathrm{e}_{\mathrm{e}} \\
\mathbf{P}_{1} \text { since } \mathrm{P}_{2} & \bullet \mathrm{P}_{1}={ }_{\mathrm{t}} \bullet \mathrm{P}_{2}, " \mathrm{Pi}=\mathrm{t}_{\mathrm{e}} \\
\mathrm{P}_{1} \text { until } \mathrm{P}_{2} & \bullet \mathrm{P}_{1}={ }_{t} \mathrm{e}_{\mathrm{s}}, \bullet \mathrm{P}_{2} \angle_{\mathrm{c}}{ }^{\circ} \mathrm{P}_{1} \\
\text { when }\left(\mathrm{e}_{1}, \mathrm{i}\right), & \\
\text { henceforth } \mathrm{P} & \left(\mathrm{e}_{1}, \mathrm{i}\right)={ }_{t} \bullet \mathrm{P},{ }^{\circ} \mathrm{P}={ }_{\mathrm{t}} \mathrm{e}_{\mathrm{e}}
\end{array}
$$

\section{Example}

We now give an example of the event-based specification of an autonomous robot system abstracted from an automatic manufacturing plant problem.

\subsection{Informal Problem Specification}

An object (partially complete product) enters the work-space of an autonomousrobot (Fig. 2). The sensors of the robot on sensing an incoming object, signals the perception node. Theperception node analyzes the signals to identify the incoming object, and passes on this information to theplanner node. Theplannernode decides about the work to be performed, records arrival of the object and the work performed, and also informs the actuator node for initiating the work. The timing requirement is that the system must initiate and complete the necessary actions within 500 time units (tus) and $\mathbf{1 0 0 0}$ tus respectively from the arrival of any object.

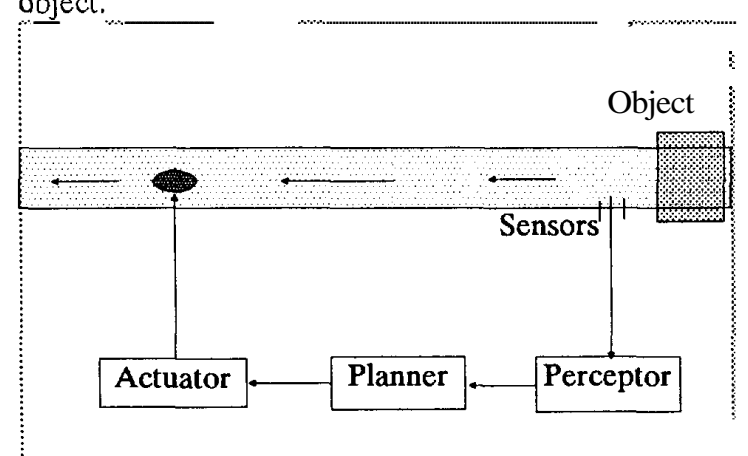

Fig. 2 Configuration of an Autonomous Robot

\section{‘2 Event-Based Specification}

Perceptor node (np) external event: np.arrival action: np.ident

Object identification. notifier event: $n$ p.nctrlr Notify Planner node.

(np.arrival,i) causesbegin(np.ident,i)

end(np.ident,i) causes (np.nctrlr,i)

Planner node (nl)

notification event: nl.objarr

action: nl.dwork

Arrival notification.

nl.update

Decide on work to be performed.

notifier event: nl.notact Record object and work performed.

(np.nctrlr,i) causes (nl.objarr,i)

(nl.objarr,i) causes begin(nl.dwork,i)

end(nl.dwork,i) causes begin(nl.update, $\mathrm{i}$ )

end(nl,dwork,i) causes (nl.notact, $\mathbf{i}$ )

Actuator node (na)

notification event: na.ntact

Notification of work action: na.actuate

(nl.notact,i) causes (na.ntact,i)

((np.ident,i);(nl,dwork,i)) fork Drive actuators.

((nl.update,i), (na.actuate,i))

(np.arrival,i) precedes begin(na.actuate,i)

by $(500,1000)$.

\section{Related Work}

The presented specification formalism has evolved from a study of the formalisms $[1,2,3,4,5,7]$. The major differencebetween our work and those reported in $[2,3]$ is that we specificallyconsider distributed real-time systems, and consider events as the basic entities of specifications. We consider nodes as the basic units of specification and explicitly consider message-passing aspects. We express the real-time behavior of distributed systems by constructing various ordering relations among the events. Our event-based specification model is in some respects similar to the event-action model of $\{1,3,5]$. However, the major difference is that unlike those in $[1,3,5]$, we use events as the basic entities of specification, and the real-time behavior is represented as relations among the events. Also, our model tries to represent distributed concurrency aspects in the tradition of Winskel's event structures. Further, our approachallows easier and more intuitiveexpression of the rich behavior of distributed real-time systems. For example, expressing the join constrict in TRIO [2] requires writing a number of formulas which obscure the intuitive understanding of the involved behavior.

\section{Conclusions and Discussions}

In this paper, we have presented a method for specification of real-time behavior of distributed systems using an event-based approach. Our specification 
method is an attempt towards the development of a formalism for specification of the real-time behavior of distributed systems $a b$ initio, rather than post hoc imposition of the timing aspects on an existing specification method. Also, we feel that an event-based approach leads to a natural specification of real-time behavior of distributed systems since events inherently represent points on a space-time diagram. Further, automatic translation of an event-based specification into equivalent specifications in a number of existing specification formalisms can save the tedium of writing complicated expressions in those formalisms. Our current work is directed towards automatically generating specificationsin a modal logic called distributed logic [8] (which we have developed specifically for specification and verification of real-time behavior of distributed systems), from event-based specifications. We are also working towards realizing an executable specification tool based on the distributed logic specifications.

\section{References}

[1] G.H. MacEwen and T.A. Montgomery, "Expressing Requirementsfor Distributed Real-TimeSystems," IEEE Computer Society Workshop on Real-Time Operating Systems, Cambridge, Massachussetts, 1987,pp. 125-128.

[2] C. Ghezzi, D. Mandrioli, and A. Morzenti,"TRIO: A Logic for Executable Specification of Real-Time Systems,"Journal of Systems and Software, Vol. 12, 1990, pp. 107-123.

[3] F. Jahanian and A. Mok, "Safety Analysis of Timing Properties of Real-Time Systems," IEEE Transactions on Software Engineering, Vol. SE-12, September 1986,pp. 890-904.

[4] G. Winskel, "An Introduction to Event Structures," in J. de Bakker, E. de Roever, and G. Rozenberg eds., Linear Time, Branching Time and Partial Order in Logics and Models of Concurrency,Springer-Verlag, Lecture Notes in Computer Science, Vol. 354, 1989.

[5] R. Mall and L.M. Patnaik, "Specification and Verification of Timing Properties of Distributed Real-time Systems," in Proc. of IEEE TENCON, Hong Kong, September 1990.

[6] L.M. Patnaik and R. Mall, "Critical Issues in RealTime Software Development," in Proc. of National Workshop on Computers in Real-Time Applications, Indore, India, February, 1991.

[7] D. Murphy, "Approachinga Real-time Concurrency Theory," in Proc. International BCS-FACS Workshop on Computing Theory, Leicester, July 1990.

[8] R. Mall and L.M. Patnaik, "A Temporal Logic over
Partial Orders for Verification of Real-timeProperties of Distributed Programs," to be presented at IEEE TENCON, Sept. 1991, New Delhi, India. 